\title{
Novel microtubule inhibitor MPT0B098 inhibits hypoxia-induced epithelial-to- mesenchymal transition in head and neck squamous cell carcinoma
}

\author{
I-Ting Tsai ${ }^{1,2+}$, Ching-Chuan Kuo ${ }^{3,4,5+}$, Jing-Ping Liou ${ }^{6}$ and Jang-Yang Chang ${ }^{1,2,4,7^{*}}$
}

\begin{abstract}
Background: Tumor hypoxia-induced epithelial-mesenchymal transition (EMT) is critical in promoting cancer metastasis. We recently discovered a novel microtubule inhibitor, MPTOB098, that employs a novel antitumor mechanism. It destabilizes hypoxia-inducible factor (HIF)-1a mRNA by blocking the function of human antigen R. Thus, we proposed that MPTOB098 modulates hypoxia-induced EMT.

Methods: In vitro IC $C_{50}$ values were determined through the methylene blue dye assay. To investigate molecular events, reverse transcriptase-polymerase chain reaction, Western blotting, immunofluorescence staining, and wound healing assay were employed.

Results: MPTOB098 significantly inhibited HIF-1a expression, epithelial-to-mesenchymal morphology changes, and migratory ability in the human head and neck squamous cell carcinoma cell line OEC-M1. Furthermore, after MPT0B098 treatment, the expression of two mesenchymal markers, vimentin and N-cadherin, was downregulated under hypoxic conditions. Moreover, MPTOB098 suppressed hypoxia-induced EMT in part by inhibiting EMT-activating transcription factors, Twist and SNAI2/Slug. In addition, the inhibition of hypoxia-induced F-actin rearrangement and focal adhesion kinase phosphorylation may have contributed to suppression of EMT by MPTOB098in OEC-M1 cells. MPTOB098 significantly inhibited transforming growth factor(TGF)- $\beta$-induced phosphorylation of receptorassociated Smad2/3 by downregulating TGF- $\beta$ mRNA and protein expression.

Conclusions: Taken together, this study provides a novel insight into the role of MPTOB098 in inhibiting hypoxia-induced EMT, suggesting its potential use for treating head and neck cancers.
\end{abstract}

Keywords: Hypoxia, Epithelial to mesenchymal transition, Microtubule inhibitor, TGF- $\beta$, Head and neck cancer

\section{Background}

In solid tumors, blood vessels form abnormally and are dysfunctional, resulting in an inability to supply sufficient oxygen and nutrients to the growing tumor mass [1]. Although a decrease in oxygen tension can be lethal for some cells, many tumor cells can survive under hypoxic conditions [2]. Tumor cells in hypoxia are resistant

\footnotetext{
* Correspondence: jychang@nhri.org.tw; z10208083@email.ncku.edu.tw ${ }^{\dagger}$ Equal contributors

${ }^{1}$ National Institute of Cancer Research, National Health Research Institutes, Tainan, Taiwan

${ }^{2}$ Institute of Molecular Medicine, College of Medicine, National Cheng Kung University, Tainan, Taiwan

Full list of author information is available at the end of the article
}

to radiation and chemotherapy; furthermore, hypoxic conditions can promote tumor progression and metastasis through numerous direct and indirect mechanisms [2]. The most critical factor responding to hypoxic conditions is hypoxia-inducible factor (HIF)- $1 \alpha$, a basic helix-loop-helix transcription factor composed of an $\alpha$ subunit (regulated by the amount of oxygen tension) and $\beta$ subunit (constitutively expressed) [3].

Epithelial-mesenchymal transition (EMT) is a cellular program in which epithelial cells lose their cell polarity and cell-cell adhesion ability and become mesenchymal cells by acquiring a fibroblastic morphology and migratory and invasive features. First recognized as a characteristic 
of embryogenic development, EMT is now indicated to be critical in tumor malignant transformation and metastasis [4]. Ample evidence indicates that hypoxia leads to characteristic changes in cell morphology that induce a mesenchymal-like phenotype and facilitate tumor cell metastasis [5].

A hypoxic microenvironment is frequently found in head and neck squamous cell carcinoma (HNSCC) and is known as a risk factor for prognosis [6, 7]. In addition, acute hypoxia can cause the development of aggressive cancers with high metastatic characteristics, resistance to chemotherapy, and higher tumor recurrence rates in patients with head and neck cancer $[6,8,9]$. We recently discovered a novel indoline-sulfonamide compound, 7aryl-indoline-1-benzene-sulfonamide (МРT0B098; Fig. 1a), that is a potent microtubule inhibitor and effective against a panel of human cancer cell lines [10]. In contrast to other clinically used microtubule inhibitors, MPT0B098 is effective in suppressing tumor growth despite p-gp170/ MDR status [11], and can destabilize HIF- $1 \alpha$ mRNA under hypoxic conditions by inhibiting the translocation of human antigen $\mathrm{R}(\mathrm{HuR})$ from the nucleus to the cytoplasm [12]. On the basis of the ability of MPT0B098 to modulate HIF-1 $\alpha$, we proposed that this compound can modulate hypoxia-induced EMT in head and neck cancers. Thus, this study investigated the effects and underlying mechanisms of MPT0B098 on hypoxia-induced EMT in the highly invasive HNSCC cell line OEC-M1.

\section{Methods}

\section{Chemicals and antibodies}

MPT0B098 was synthesized by Prof. Jing-Ping Liou at the College of Pharmacy, Taipei Medical University, Taipei, Taiwan. The detailed synthetic procedure was described previously [11]. Colchicine and paclitaxel were purchased from Sigma-Aldrich (St. Louis, MO). Monoclonal antibodies for Smad2, phospho-Smad2 (Ser465/ 467), Smad3, phospho-Smad3, Smad2/3, and SNAI2/Slug and polyclonal antibodies for transforming growth factor (TGF)- $\beta$, focal adhesion kinase (FAK), and phospho-FAK were purchased from Cell Signaling Technology (Danvers, MA). Monoclonal antibodies for HIF- $1 \alpha$ and N-cadherin were purchased from BD Biosciences (San Jose, CA). A polyclonal antibody for vimentin was purchased from Santa Cruz Biotechnology (Santa Cruz, CA). A polyclonal antibody for Twist was purchased from GeneTex (Irvine, CA). A monoclonal antibody for GAPDH was purchased from Merck Millipore (Darmstadt, Germany). All other chemicals of standard analytic grade or higher were purchased from E. Merck Co. (Darmstadt, Germany) or Sigma-Aldrich (St. Louis, MO).

\section{Cell lines and cell culture}

OEC-M1 cells, derived from a human oral epidermoid carcinoma, were established and provided by Dr. ChingLiang Meng from the Department of Dentistry, TriService General Hospital, National Defense Medical Center, Taipei, Taiwan [13]. The cells were cultured in RPMI 1640 medium, supplemented with $10 \%$ fetal bovine serum in a humidified $5 \% \mathrm{CO}_{2}$ incubator at $37{ }^{\circ} \mathrm{C}$. For hypoxic conditions, cells were incubated in a hypoxic chamber with a gas mixture of $1 \% \mathrm{O}_{2}$ and $5 \% \mathrm{CO}_{2}$ balanced with nitrogen.

\section{Cell morphology assay}

Cells were stained with $0.5 \%$ crystal violet in 95\% ethanol for $1 \mathrm{~h}$ and then examined through light microscopy.

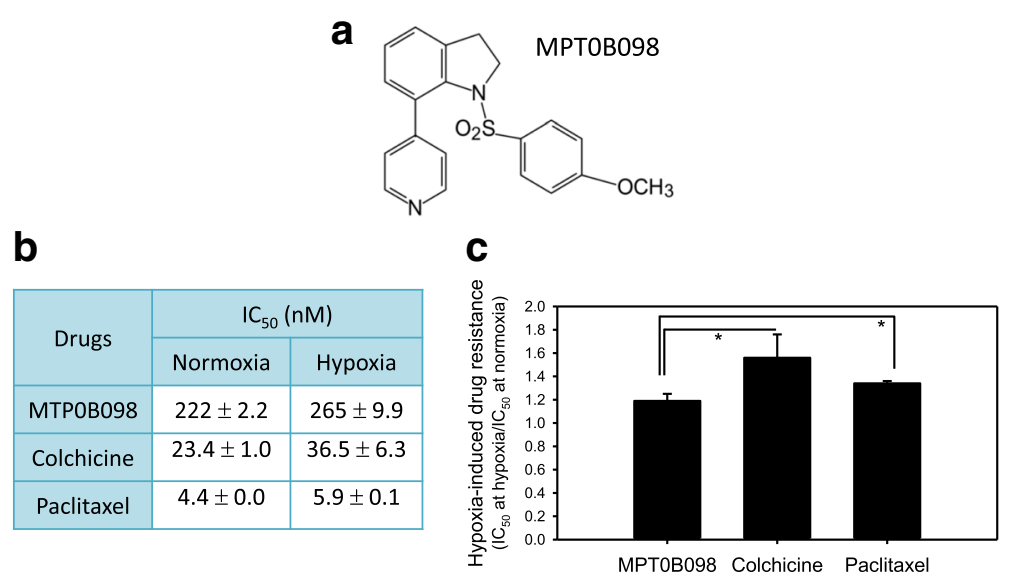

Fig. 1 Antiproliferative effect of MPTOB098 in OEC-M1 cells under normoxic and hypoxic conditions. a The chemical structure of MPTOB098. b The in vitro antiproliferative activity of MPTOB098 in OEC-M1 cells under normoxic and hypoxic conditions. OEC-M1 cells were treated with MPTOB098, colchicine, or paclitaxel under normoxic and hypoxic conditions for $72 \mathrm{~h}$. The $\mathrm{IC}_{50}$ values of these compounds resulting from $50 \%$ inhibition of cell growth were calculated using the methylene blue dye assay. Each value represents the mean \pm SD of three independent experiments. c Hypoxia-induced drug resistance is the $\mathrm{IC}_{50}$ value of the test compounds in hypoxia divided by the equivalent in normoxia $\left({ }^{*} p<0.05\right)$ 


\section{Cell viability assay}

Cells in a logarithmic growth phase were cultured at a density of 10,000 cells per well in a 24-well plate. The cells were exposed to various concentrations of the test drug for $72 \mathrm{~h}$. The methylene blue dye assay [14] was used to evaluate drug effect on cell growth. The $\mathrm{IC}_{50}$ values resulting from $50 \%$ inhibition of cell growth were calculated graphically, compared with control group growth.

\section{Western blotting}

Cells were initially seeded at a density of $4 \times 10^{5}$ cells in $100-\mathrm{mm}^{2}$ dishes. Following treatment, cell pellets were collected and lysed in a buffer $(50 \mathrm{mM}$ Tris- $\mathrm{HCl}, \mathrm{pH} 8$. $0,150 \mathrm{mM} \mathrm{NaCl}, 5 \mathrm{mM}$ EDTA, $2 \mathrm{mM}$ dithiothreitol, $2 \mathrm{mM} \mathrm{Na}_{3} \mathrm{VO}_{4}, 0.25 \mathrm{mM}$ PMSF, $10 \mathrm{mM} \mathrm{NaF}, 0.5 \% \mathrm{NP}-$ 40 , and $20 \mu \mathrm{g} / \mathrm{mL}$ each of aprotinin, leupeptin, and pepstatin [proteinase inhibitors]). The supernatants were collected and the amount of protein was quantified. Equal amounts of protein from each lysate were separated by SDS-PAGE, blotted on polyvinylidenedifluoride membranes, conjugated with various specific primary antibodies, and then probed with appropriate secondary antibodies. The immunoreactive bands were detected using the enhanced chemiluminescent method and then visualized on Kodak Bio-MAX MR film.

\section{Wound healing assay}

Cells were grown to $80 \%$ confluence on culture dishes; then, a wound was created in the center of the cell monolayer with a plastic pipette tip. The migration of cells into the wound area was assessed after 4, 6, 8, and $18 \mathrm{~h}$ at $37{ }^{\circ} \mathrm{C}$ under normoxic $\left(5 \% \mathrm{CO}_{2}, 21 \% \mathrm{O}_{2}\right)$ and hypoxic $\left(5 \% \mathrm{CO}_{2}, 1 \% \mathrm{O}_{2}\right.$, balanced with $\mathrm{N}_{2}$ ) conditions. The area of denuded surface was quantified immediately and at the time points of 4, $6,8 \mathrm{~h}$ after wounding. The extent of wound closure was determined by calculating the ratio between the surface area of the wound for each time point and the surface of the initial wound.

\section{Immunofluorescence analysis of F-actin}

OEC-M1 cells were fixed with 4\% paraformaldehyde (Electron Microscopy Sciences, Hatfield, PA), permeabilized with $0.5 \%$ Triton X-100 in phosphate-buffered saline, treated with $0.1 \%$ sodium borohydride (Sigma-Aldrich, St. Louis, $\mathrm{MO}$ ), blocked with $5 \%$ horse serum, and then incubated with phalloidin (Life Technologies, Gaithersburg, MD) for $30 \mathrm{~min}$ at room temperature in the dark. Nuclei were stained with DAPI. Cells were observed with an OLYMPUS fluorescence microscope.

Reverse transcriptase-polymerase chain reaction analysis Ten micrograms of total RNA, extracted with Trizol reagent (Life Technologies, Gaithersburg, MD), were treated with DNase and converted to cDNA using the SuperScript II RNase H Reverse Transcriptase System (Invitrogen, Carlsbad, CA). Reverse transcriptase-poly merase chain reaction (RT-PCR) was performed using a Perkin-Elmer GeneAmp PCR System 2400 (Applied Biosystems, Foster City, CA). PCR primers and TaqMan probes (5'-GACCTGGA-3') used to amplify the indicated genes were designed using Primer Express (version 1.0; Applied Biosystems) as follows: TGF- $\beta 1$ forward 5'cggagttgtgcggcagtggttga- $3^{\prime}$ and reverse $5^{\prime}$-ggcgcccgggtt atgctggttgta- $3^{\prime}$ and TGF- $\beta 2$ forward $5^{\prime}$-gtcttggatgcggect attgc- $3^{\prime}$ and reverse $5^{\prime}$-gctgcatttgcaagactttac- $3^{\prime}$. The reaction mixture was preheated at $95{ }^{\circ} \mathrm{C}$ for $5 \mathrm{~min}$, followed by 30 cycles of $95{ }^{\circ} \mathrm{C}$ for $30 \mathrm{~s}, 55{ }^{\circ} \mathrm{C}$ for $30 \mathrm{~s}$, and $72{ }^{\circ} \mathrm{C}$ for $40 \mathrm{~s}$; final extension was at $72{ }^{\circ} \mathrm{C}$ for $7 \mathrm{~min}$. In a separate reaction, GAPDH was amplified as the reference gene. PCR products of the target genes were analyzed through electrophoresis on $2 \%$ agarose gel and visualized using ethidium bromide staining under UV light. Quantitative data was collected by measuring the bands using ImageJ (Bethesda, MD).

\section{Statistical analysis}

Quantitative data were reported as mean \pm SD. Statistical calculations were completed on Microsoft Excel. $P$ values for determining statistical significance were calculated using an unpaired two-tailed Student's $t$ test.

\section{Results}

MPT0B098 exhibits low-level resistance toward OEC-M1 cell growth under hypoxic conditions

We used the methylene blue dye assay to examine the antiproliferative efficacy of MPT0B098 and other clinically used microtubule inhibitors, such as colchicine and paclitaxel, in OEC-M1 cells. As shown in Fig. 1b, MPT0B098 inhibited the growth of OEC-M1 cells with $\mathrm{IC}_{50}$ of 222 and $265 \mathrm{nM}$ under normoxic and hypoxic conditions, respectively. This result indicates that hypoxia leads to increased low-level drug resistance of MPT0B098 in OEC-M1 cells (Fig. 1c).

In addition, compared with MPT0B098, other microtubule inhibitors, including colchicine and paclitaxel, exhibited higher resistance in OEC-M1 cells under hypoxic conditions than under normoxic conditions. The $\mathrm{IC}_{50}$ values of colchicine were 23 and $37 \mathrm{nM}$ under normoxia and hypoxia, respectively, and the $\mathrm{IC}_{50}$ values of paclitaxel were 4.4 and $5.9 \mathrm{nM}$, respectively (Fig. 1b). These results indicate that МРТ0В098 is more effective in overcoming hypoxia-induced drug resistance than colchicine and paclitaxel in OEC-M1 cells.

MPTOB098 inhibits hypoxia-induced EMT in OEC-M1 cells Intratumoral hypoxia induces EMT and promotes cancer metastasis. HIF- $1 \alpha$ plays a critical role in driving the 
characteristic changes in cell morphology causing a mesenchymal-like phenotype and facilitating the metastasis of tumor cells $[5,15]$. Because MPT0B098 can inhibit HIF- $1 \alpha$ mRNA and protein expression in the human lung adenocarcinoma cell line A549 [12], we speculated that this compound inhibits HIF- $1 \alpha$ expression and suppresses EMT in OEC-M1 cells. Consistent with our previous findings, MPT0B098 demonstrated potent inhibition of HIF- $1 \alpha$ expression in a concentration-dependent manner under hypoxic conditions in OEC-M1 cells (Fig. 2a and b). In addition, the inhibitory effect of MPT0B098 on HIF- $1 \alpha$ was found in another human HNSCC cell line, SCC-15 (Additional file 1: Figure S1).

On further examining the role of МРТ0В098 in hypoxia-induced EMT in OEC-M1 cells, we found that OEC-M1 cells displayed epithelial characteristics under normoxic conditions, with a round morphology and linked cells (Fig. 2c, left panel). However, under hypoxic conditions, cells displayed a fibroblastic morphology and lost their cell-cell contact, which is a mesenchymal characteristic, implying that hypoxia can trigger EMT (Fig. 2c, middle panel). Notably, the transformation from epithelial to mesenchymal cell type under hypoxic conditions could be inhibited by treating cells with MPT0B098 (Fig. 2c, right panel). These results suggest that MPT0B098 may play a role in modulating hypoxiainduced EMT in OEC-M1 cells.

\section{MPT0B098 is more potent in inhibiting hypoxia-induced mesenchymal marker expression than clinically used microtubule inhibitors}

MPT0B098 could inhibit the expression of hypoxiainduced mesenchymal markers, including vimentin and $\mathrm{N}$-cadherin, in a concentration-dependent manner in OEC-M1 cells (Fig. 3a and 3b). Because MPT0B098 inhibited hypoxia-induced EMT, we analyzed whether other clinically used microtubule inhibitors, such as colchicine and paclitaxel, also suppress vimentin and $\mathrm{N}$ cadherin expression in OEC-M1 cells under hypoxic conditions. MPT0B098 caused a reduction in vimentin and $\mathrm{N}$-cadherin expression in a concentration dependent manner, but the same effect was not observed in colchicine- or paclitaxel-treated cells (Fig. 3a and 3b).

\section{MPT0B098 suppresses hypoxia-induced EMT partially by inhibiting the expression of EMT-activating transcription factors Twist and SNAI2/slug}

During EMT, molecular reprogramming is triggered and orchestrated by various EMT-activating transcription factors, including Twist and SNAI2/Slug [16]. As shown
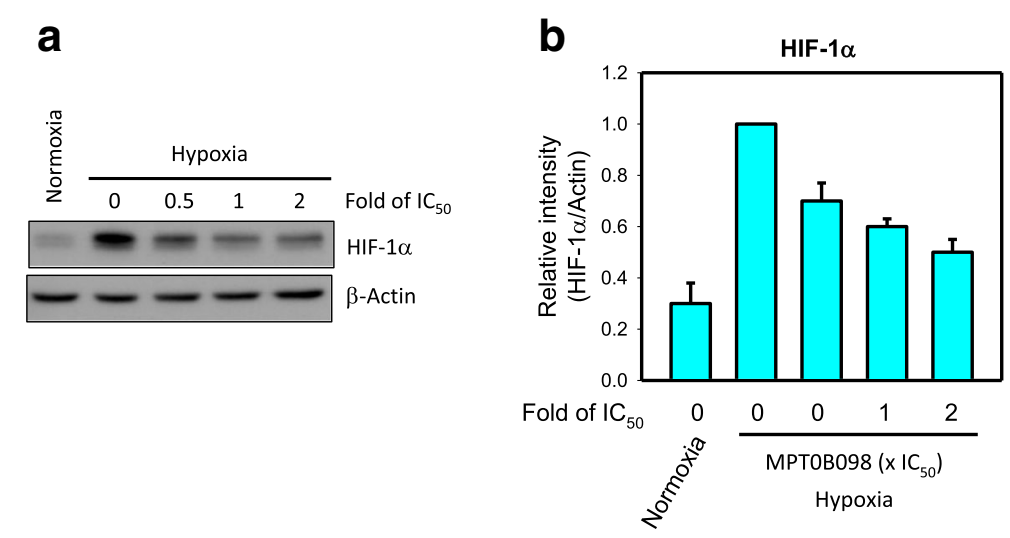

C
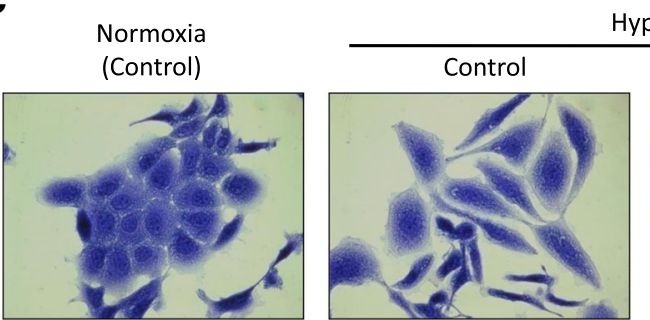

ypoxia

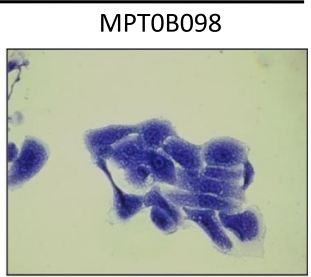

Fig. 2 MPTOB098 inhibits hypoxia-induced EMT in OEC-M1 cells. a The effect of MPT0B098 onhypoxia-induced HIF-1aexpression. OEC-M1 cells were treated with various concentrations, indicated as fold of $\mathrm{IC}_{50}$ values, of MPTOB098 for $18 \mathrm{~h}$ under hypoxic conditions. At the end of the drug treatment, cell lysates were prepared and analyzed by SDS-PAGE and Western blot. $\beta$-Actin was used as an internal control. b Each bar depicts the mean of the relative intensity of HIF-1a from three independent experiments. c The effect of MPTOB098 on hypoxia-induced EMT.Cells were treated with MPTOB098 at a concentration of 0.5 -fold $\mathrm{IC}_{50}$ for $48 \mathrm{~h}$ under hypoxic conditions and then cell morphology was examined by crystal violet staining. Cells in normoxia were used as controls 

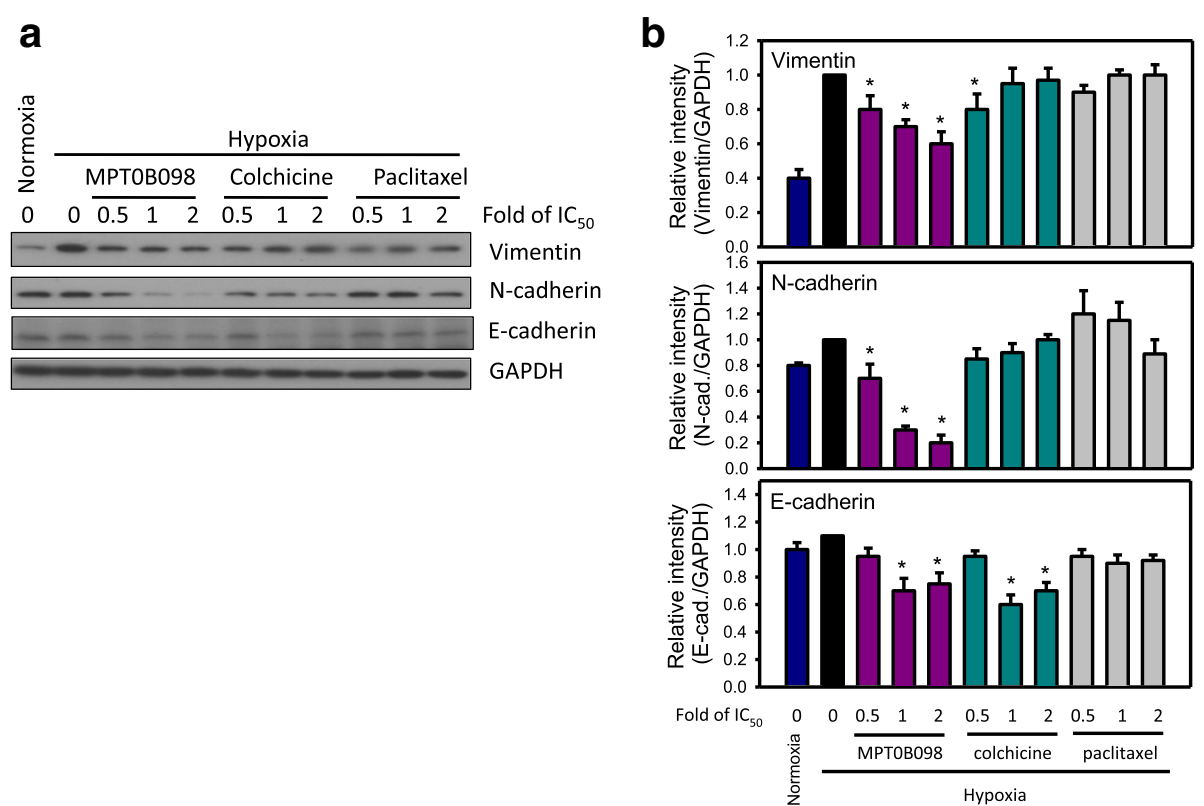

Fig. 3 Effect of MPTOB098 and other microtubule inhibitors on the expression of EMT-related proteins in OEC-M1 cells under hypoxic conditions. a Parallel comparison of the effects of MPTOB098, colchicine, and paclitaxel on the expression of vimentin, N-cadherin, and E-cadherin in OEC-M1 cells.OEC-M1 cells were treated with various concentrations,indicated as fold of $\mathrm{IC}_{50}$ values, Of MPTOB098, colchicine, or paclitaxel for $36 \mathrm{~h}$ under hypoxic conditions. At the end of the drug treatment, cell lysates were prepared and analyzed by SDS-PAGE and Western blot. GAPDH was used as an internal control. $\mathbf{b}$ Each bar depicts the mean of the relative intensity of vimentin, $\mathrm{N}$-cadherin, and E-cadherin from three independent experiments (* $p<0.05$, compared to hypoxia control).

in Fig. 4a and b, we found that the expression levels of Twist and SNAI2/Slug were significantly suppressed in a concentration-dependent manner when cells were treated with various concentrations of MPT0B098 under hypoxic conditions for 18 and $36 \mathrm{~h}$. These results indicate that MPT0B098 suppresses hypoxia-induced EMT partially by inhibiting the expression of EMT-activating transcription factors, Twist and SNAI2/Slug, in OEC-M1 cells.

\section{MPT0B098 significantly inhibits hypoxia-induced F-actin rearrangement and FAK phosphorylation}

Actin filaments (F-actin) are organized in thin bundles in epithelial cells; however, in trans-differentiated mesenchymal cells, they are bundled into thick contractile stress fibers at the cell surface. Thus, F-actin rearrangement is associated with increased cell movement during EMT; it is also required for metastatic cancer cells to
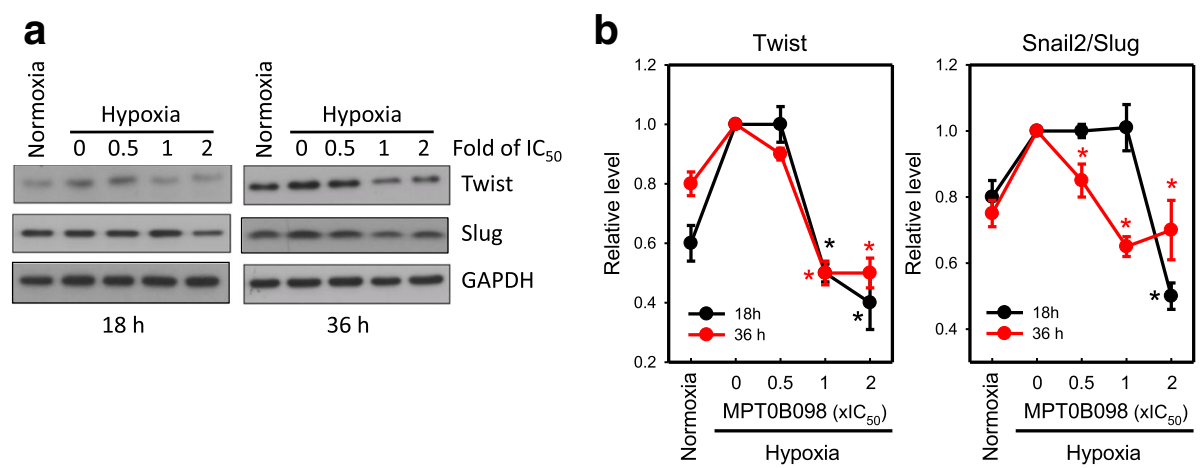

Fig. 4 MPTOB098 suppresses hypoxia-induced EMT by inhibiting the expression of EMT-activating transcription factors, Twist and SNAI2/Slug. a The effects of MPTOB098 on the expression of Twist and SNAI2/Slug in OEC-M1 cells.OEC-M1 cells were treated with various concentrations, indicated as fold of $\mathrm{IC}_{50}$ values, of MPTOB098 for 18 and $36 \mathrm{~h}$ under hypoxic conditions. At the end of the drug treatment, cell lysates were prepared and analyzed by SDS-PAGE and Western blot. GAPDH was used as an internal control. Each values depicts the mean of the relative intensities of Twist and SNAI2/Slug from three independent experiments. $\mathbf{b}$ Each value depicts the mean of the relative intensities of Twist and SNAI2/Slug from three independent experiments ( ${ }^{*} p<0.05$, compared to hypoxia control) 
spread from primary tumors $[17,18]$. As shown in Fig. 5a, f-actin is located within the cytoplasm under normoxic conditions. By contrast, hypoxia-induced Factin rearrangement showing increased expression of stress fiber patterns and concentrated at the plasma membrane on the edge of the cells. MPT0B098-treated OECM1 cells demonstrated a reduction in the expression of hypoxia-induced stress fiber patterns and membrane localization of F-actin in a concentration-dependent manner.

FAK is a cytoplasmic tyrosine kinase, with critical roles in the regulation of cell spreading, migration, and survival and in the downstream signaling cascade associated with its own phosphorylation $[19,20]$. Changes in the organization of the actin cytoskeleton, influenced by FAK, leads to remarkable changes in the tyrosine phosphorylation of several signaling proteins localized at the focal adhesion complex [21]. To further explore the molecular mechanisms responsible for MPT0B098 inhibition of hypoxia-induced actin cytoskeletal rearrangement, we examined the expression and activation of FAK protein. As shown in Fig. 5b and c, FAK expression and activation were increased under hypoxic conditions. Hypoxia-induced phosphorylation of FAK decreased when the hypoxic cells were treated with MPT0B098. Taken together, these results suggest that МРТ0В098- inhibited FAK/actin cytoskeletal rearrangement partially contributes to EMT suppression.

\section{MPT0B098 downregulates TGF- $\beta$-induced phosphorylation of receptor-associated Smads}

The reprogramming of gene expression during EMT is initiated and controlled by signaling pathways responding to extracellular cues, including TGF- $\beta$, fibroblast growth factor, epidermal growth factor, hepatocyte growth factor, Wnt/ $\beta$-catenin, and Notch [22]. Among these, TGF- $\beta$ signaling has a dominant role in the initiation of EMT programs that develop cancer progression $[16,22,23]$. We explored whether the TGF- $\beta$ signaling pathway is involved in MPT0B098-mediated inhibition of hypoxia-induced EMT. Our results indicated that MPT0B098 suppressed the phosphorylation of Smad2 and Smad3 in hypoxia in OEC-M1 cells (Fig. 6a and b). In addition, MPT0B098 inhibited Smad signaling in another human HNSCC cell line, SCC-15 (Additional file 1: Figure S1).

To further examine the involvement of TGF- $\beta$ in the hypoxia-induced activation of Smad signaling, TGF- $\beta$ was applied to MPT0B098-treated hypoxic cells. TGF- $\beta$ treatment significantly enhanced Smad2 and Smad3 phosphorylation even under hypoxic conditions (Fig. $6 \mathrm{c}$ and d). Coadministration of TGF- $\beta$

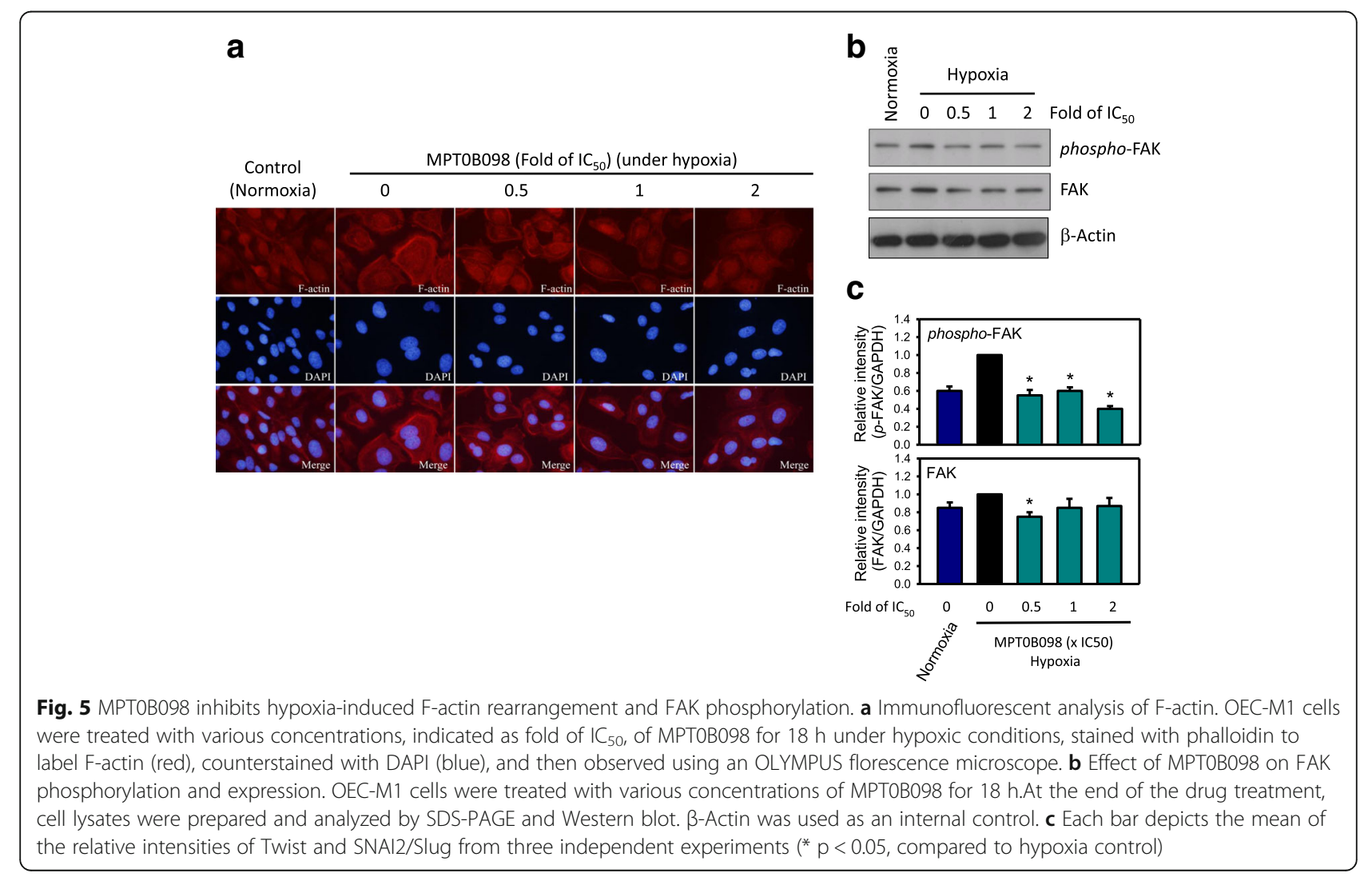


a

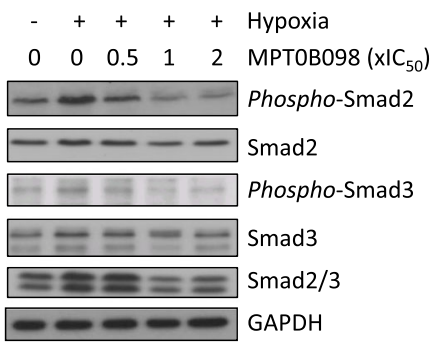

b
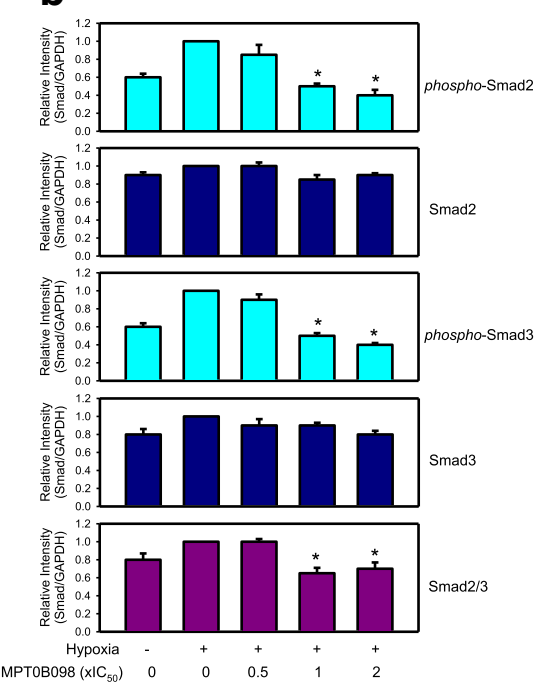

C

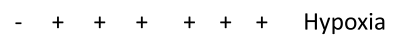

-++++- TGF- $\beta$

$\begin{array}{llllllll}0 & 0 & 0 & 0.5 & 1 & 2 & 2 & \text { MPTOB098 }\left(x \mid C_{50}\right)\end{array}$

$-\infty-\infty$ Phospho-Smad2

$---\cdots-\infty$ Smad2

Phospho-Smad3

- $-\ldots-\ldots$ Smad3

$=-\ldots=\ldots \operatorname{Smad} 2 / 3$

$----\infty$ GAPDH

d

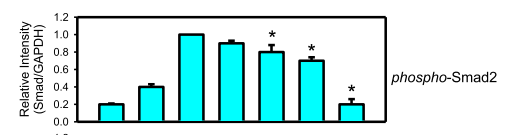

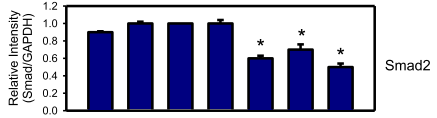

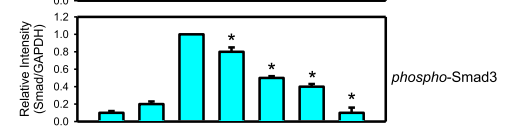

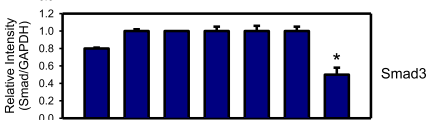

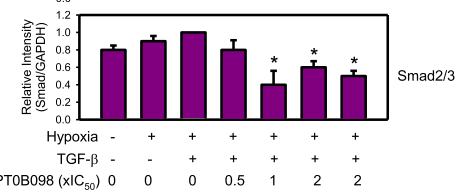

Fig. 6 MPTOB098 downregulatesTGF- $\beta / S m a d$ signalingin OEC-M1 cells. a Cells were treated with various concentrations,indicated as fold of IC $C_{50}$ values, of MPTOB098 for $36 \mathrm{~h}$ under hypoxic conditions. $\mathbf{b}$ Each bar depicts the mean of the relative intensities of phospho-Smads and Smads from three independent experiments ( ${ }^{*} \mathrm{p}<0.05$, compared to hypoxia control). c Cells were treated with various concentrations,indicated as fold of $\mathrm{C}_{50}$ values, of MPTOB098 for $18 \mathrm{~h}$, followed by an addition of $5 \mathrm{ng} / \mathrm{mL}$ TGF- $\beta$ for another $18 \mathrm{~h}$ under hypoxic conditions. At the end of the drug treatment, cell lysates were prepared and analyzed by SDS-PAGE and Western blot. GAPDH was used as an internal control. $\mathbf{d}$ Each bar depicts the mean of the relative intensities of phospho-Smads and Smads from three independent experiments ( $\mathrm{p}<0.05$, compared to hypoxia control)

and MPT0B098 significantly suppressed Smad2 and Smad3 phosphorylation in a concentration-dependent manner. In addition, MPT0B098 slightly inhibited Smad2 and Smad3 protein expression (Fig. 6c and d), suggesting that MPT0B098 influenced Smad inactivation under hypoxic conditions through TGF- $\beta$ modulation.

\section{MPTOB098 downregulates TGF- $\beta$ signaling by decreasing expression levels of TGF- $\beta$ mRNA and protein}

To further explore the relationship between MPT0B098 and TGF- $\beta$ signaling, we examined the expression levels of TGF- $\beta$ mRNA and protein in MPT0B098-treated hypoxic cells. We found that MPT0B098 suppressed TGF- $\beta$ mRNA (Fig. 7a) and protein expression (Fig. 7b) in a dose-dependent manner under hypoxic conditions in OEC-M1 cells. In addition, we found that MPT0B098 inhibited TGF- $\beta$ protein expression under normoxic conditions (Fig. 7c). These results suggest that MPT0B098 regulates the EMT process in part through a reduction in
TGF- $\beta$ mRNA and protein levels followed by downregulation of the TGF- $\beta /$ Smad signaling cascades.

\section{MPTOB098 inhibits hypoxia-induced cell migration in OEC-M1 cells}

Since EMT is associated with tumor migration, especially under hypoxic conditions [4, 24], we investigated whether MPT0B098 inhibits the EMT program, which then leads to suppression of the migratory capability of OEC-M1 cells in hypoxia. In the wound healing assay we observed that hypoxia induces greater cell migration compared with normoxia, whereas MPT0B098 inhibits hypoxia-induced cell migration with $8 \mathrm{~h}$ treatment in OEC-M1 cells (Fig. 8a). From quantification of the wounded area we found that MPT0B098 effectively inhibited hypoxic cell migration in a time-dependent manner (Fig. 8b, left panel), without impairing cell viability (Fig. 8b, right panel), compared with control cells with a treatment duration of 4-18 h. Moreover, we 
a

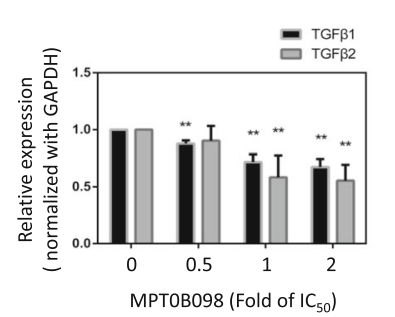

b
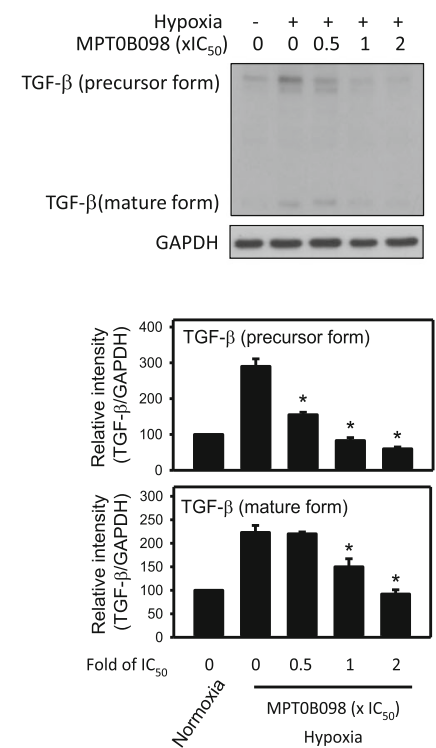

C
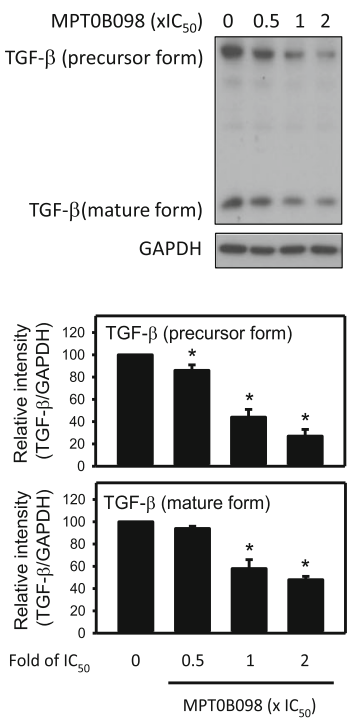

Fig. 7 MPTOB098 downregulates TGF- $\beta$ signaling by decreasing the expression of TGF- $\beta$ mRNA and protein in OEC-M1 cells under hypoxic conditions. a Effect of MPTOB098 on the expression levels of TGF- $\beta 1$ and TGF- $\beta 2$ mRNA. Cells were treated with various concentrations of MPTOB098 in hypoxia. After incubation for $36 \mathrm{~h}$, total RNA was extracted, reverse transcribed into CDNA, and subjected to PCR for detection of TGF- $\beta 1$ and TGF- $\beta 2$. GAPDH was used as an internal control. Data are represented as mean \pm SD in triplicate. ${ }^{* *} P<0.01$ and ${ }^{* *} P<0.001$ for comparison between the control and treatment groups using an unpaired two-tailed Student's t test. $\mathbf{b}$ Effect of MPT0B098 on the expression level of TGF- $\beta$ protein. Cells were treated with various concentrations of MPTOB098 for $36 \mathrm{~h}$ in hypoxia. Quantification of TGF- $\beta$ protein was determined by normalization with GAPDH (* $p<0.05$, compared to hypoxia control). c The inhibitory effect of MPTOB098 on TGF- $\beta$ under normoxic conditions. Cells were treated with MPTOB098 in normoxia at the indicated concentrations for $36 \mathrm{~h}$. Quantification of TGF- $\beta$ protein was determined by normalization with GAPDH (* $p<0.05$, compared to control)

compared the antimigratory effect of MPT0B098 with other microtubule inhibitors, including colchicine and paclitaxel, and found that the wound healing inhibitory activity of MTP0B098 was significantly higher than colchicine and paclitaxel at a drug concentration of 0.5 -fold $\mathrm{IC}_{50}$, under hypoxic conditions (Fig. 8c).

\section{Discussion}

Microtubule-targeting drugs, such as taxotere, epothilone $\mathrm{B}$, discodermolide, vincristine, 2-methoxyestradiol, and colchicine, can downregulate the expression of HIF$1 \alpha$ protein but not HIF-1 $\alpha$ mRNA [25]. MPT0B098, a novel indoline-sulfonamide-based microtubule inhibitor, can destabilize HIF-1 $\alpha$ mRNA in hypoxia by inhibiting the translocation of HuR from the nucleus to cytoplasm [12]. This ability distinguishes MPT0B098 from other microtubule inhibitors. MPT0B098 is effective against a panel of human cancer cell lines, regardless of the pgp170/MDR status [10-12]. Because MPT0B098 has a unique ability to modulate HIF- $1 \alpha$, we proposed that MPT0B098 also suppresses hypoxia-induced malignant transformation.

Hypoxia can induce EMT and enhance the migration and invasion ability of tumor cells [26]. EMT is a key step for tumor metastasis. Hypoxia leads to characteristic changes in cell morphology, causing a mesenchymal-like phenotype, a distinct characteristic of the EMT process [4]. In this study, we selected a highly invasive human HNSCC cell line, OEC-M1 [27], to clarify the role of MPT0B098 in EMT regulation. We observed that MPT0B098 could inhibit expression of HIF- $1 \alpha$ protein and the hypoxiainduced mesenchymal-like phenotype in OEC-M1 cells (Fig. 2c). In addition, this compound was more effective, as indicated by low hypoxia-induced drug resistance, than colchicine and paclitaxel in inhibitingOEC-M1 cell growth under hypoxic conditions (Fig. 1c). As determined by expression of the two mesenchymal markers vimentin and $\mathrm{N}$-cadherin, we noted that MPT0B098 was more potent in inhibiting hypoxia-induced vimentin and N-cadherin upregulation than colchicine and paclitaxel in OEC-M1 cells (Fig. 3). These results suggest that MPT0B098 is distinct from other clinically used microtubule inhibitors in its inhibition of hypoxia-induced EMT. Unlike the classic reversal of the EMT phenomenon, E-cadherin protein was slightly suppressed by MPT0B098 (Fig. 3a and 3b). Cadherins have been reported to regulate the organization and dynamics of microtubules. This behavior may also affect cadherin biology through microtubule-based vesicular traffic [28]. Thus, the interplay between E-cadherin and microtubules may be disrupted by the inhibition of microtubule 


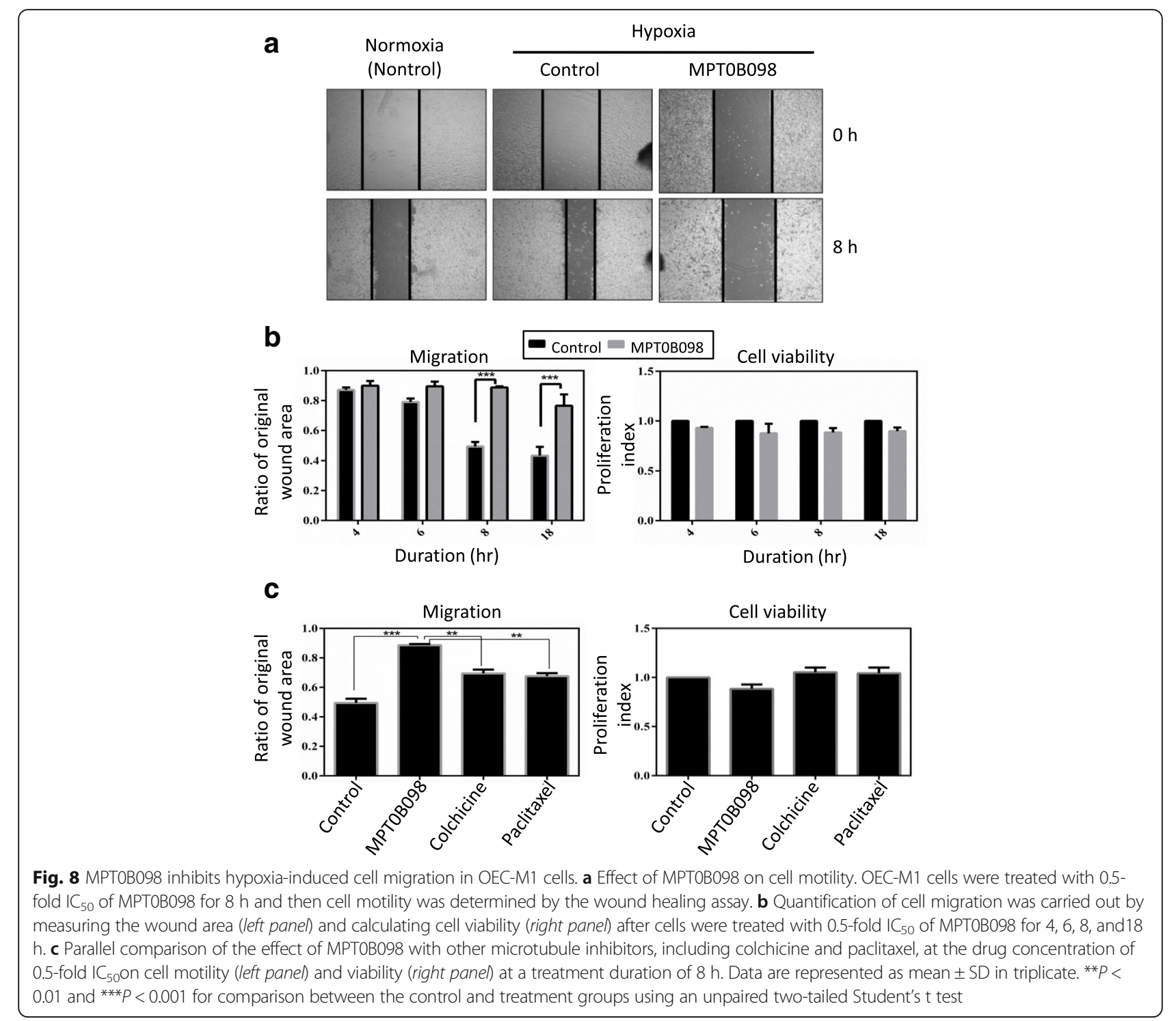

polymerization under MPT0B098 treatment. The underlying mechanisms merit further investigation.

Molecular reprogramming occurring during EMT is triggered and orchestrated by various EMT-activating transcription factors, including Twist and SNAI2/Slug [16]. Yang et al. found that HIF- $1 \alpha$ regulated the expression of Twist by binding directly to the hypoxiaresponse element in the Twist proximal promoter. In addition, silencing of Twist in HIF-1 $\alpha$-overexpressing or hypoxic cells reversed EMT and metastatic phenotypes [29]. Cheng et al. reported that treatment with HIF- $1 \alpha$ siRNA diminished the upregulation of SNAI2/ Slug expression [30]. Because MPT0B098 is effective in suppressing HIF- $1 \alpha$ expression, we investigated the effect of MPT0B098 on modulation of Twist and SNAI2/ Slug and found that the expression levels of hypoxia- induced Twist and SNAI2/Slug were significantly decreased in a concentration-dependent manner when cells were treated with various concentrations of MPT0B098 (Fig. 4).

Transcription program switching in EMT is induced by signaling pathways mediated by TGF- $\beta$, bone morphogenetic protein, Wnt- $\beta$-catenin, Notch, Hedgehog, and receptor tyrosine kinases. These pathways are activated by various dynamic stimuli from the local microenvironment. Of note, TGF- $\beta$ signaling has a dominant role in EMT [22, 30-32]. Here, we demonstrated that MPT0B098 inhibits hypoxia-induced EMT by downregulating the expression levels of TGF- $\beta$ mRNA (Fig. 7a) and protein (Fig. 7b). We previously demonstrated that MPT0B098 inhibited HuR translocation to the cytoplasm [12]. HuR, an mRNA-stabilizing protein, 


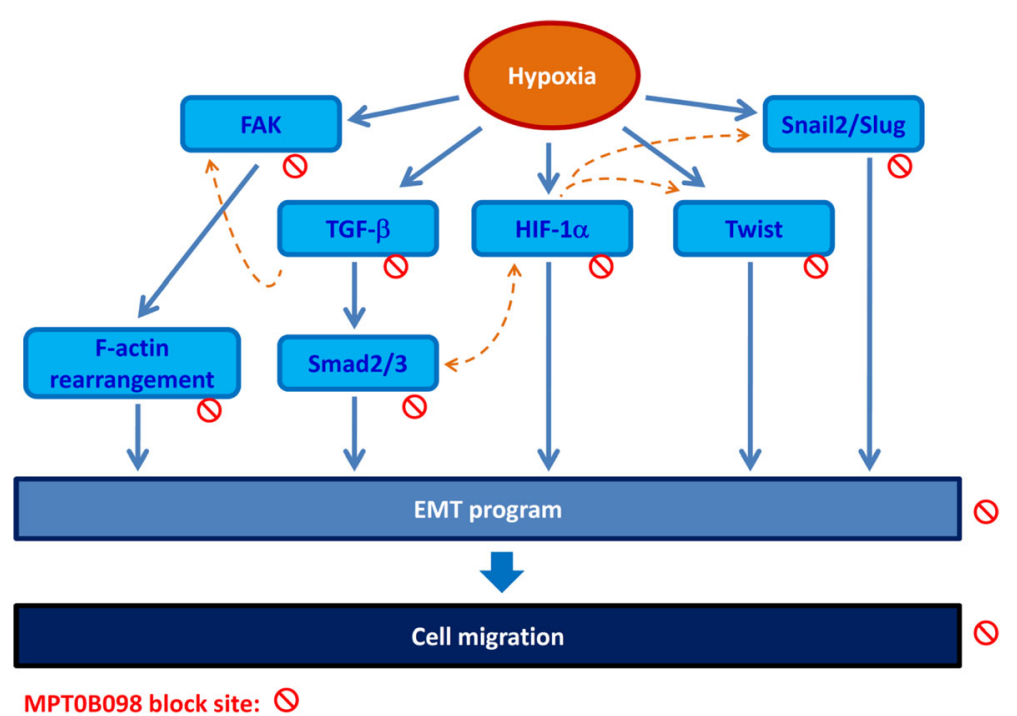

Fig. 9 Proposed pathway of MPTOB098-mediated EMT suppression. Blue solid line: proposed working models; orange dotted line: literature reported

shuttles between the nucleus and cytoplasm through several export pathways. Mobilizing $\mathrm{HuR}$ from the nucleus to the cytoplasm leads to maintenance of the stability of HuR-mediated target mRNAs. Increased stability of TGF$\beta$ mRNA by HuR promotes EMT in pancreatic cancer [33]; therefore, whether МРТ0В098 affect the stability of TGF- $\beta$ mRNA through HuR dependent or independent mechanisms which needs to be further investigated.

TGF- $\beta$ signaling toward EMT is mediated by both Smad-dependent and -independent pathways. The Smad pathway is unique to TGF- $\beta$ signaling [34]. Our further evaluation demonstrated that MPT0B098 inhibited hypoxia-induced EMT by blocking TGF- $\beta$-dependent Smad signaling (Fig. 6). Because TGF- $\beta$ signaling occurs not only in hypoxia but also in normoxia, we found that MPT0B098 decreased the expression of TGF- $\beta$ protein under normoxic conditions (Fig. 7c), suggesting that the inhibitory effect of MPT0B098 on TGF- $\beta$ signaling was not restricted to the hypoxic condition. Zhang et al. reported that exposure of cells to hypoxia resulted in phosphorylation of Smad2 and Smad3 as well as stimulation of transcriptional activities of HIF- $1 \alpha$ and upregulation of TGF- $\beta 2$ expression, suggesting that autocrine regulation of TGF- $\beta 2$ production in hypoxia may involve crosstalk between Smad3 and HIF-1 $\alpha$ signaling pathways [35]. The interplay between each molecule in response to MPT0B098 needs further elucidation. In addition to TGF- $\beta /$ Smad signaling, Cicchini et al. reported that TGF- $\beta$ induces a Src-dependent activation of FAK protein [36]. The results shown in Fig. 5b show that MPT0B098 significantly suppressed hypoxia-induced FAK phosphorylation. Because FAK is a critical modulator in regulating actin cytoskeleton organization [19-21], we further observed that MPT0B098 inhibited hypoxiainduced expression of the stress fiber pattern and membrane localization of F-actin (Fig. 5a). Accordingly, we proposed that MPT0B098 inhibits hypoxia-induced EMT in HNSCC by (1) suppressing HIF-1 $\alpha$ expression, (2) inhibiting the EMT-activating transcription factors Twist and SNAI2/Slug, (3) blocking TGF- $\beta /$ Smad signaling, and (4) interfering with FAK-mediated actin cytoskeleton rearrangement (Fig. 9). Further evaluation to clarify the interplay between MPT0B098 and the particular molecules is warranted.

\section{Conclusions}

Taken together, we demonstrated for the first time that the novel microtubule Inhibitor MPT0B098 inhibits hypoxia-induced EMT in HNSCC through modulation of HIF- $1 \alpha$, Twist, SNAI2/Slug, TGF- $\beta /$ Smad signaling, and FAK/actin cytoskeleton rearrangement. The results presented here may provide novel insight into the mechanism of action of microtubule inhibitors for inhibiting EMT. MPT0B098 has great potential for clinical treatment of hypoxic tumors and merits further investigation.

\section{Additional file}

Additional file 1: Supplemental materials. (DOCX $739 \mathrm{~kb}$ )

\section{Abbreviations}

EMT: epithelial-mesenchymal transition; FAK: focal adhesion kinase;

GAPDH: glyceraldehyde 3-phosphate dehydrogenase; HIF-1a: hypoxiainduced factor 1 alpha; HNSCC: head and neck squamous cell carcinoma; HuR: human antigen R; TGF- $\beta$ : transforming growth factor beta 


\section{Acknowledgements}

All authors sincerely acknowledge Ms. Hsin-Yi Pan for assisting graphics processing, and thank the funding support.

\section{Funding}

This study was supported by following grants from the following agencies: the Ministry of Health and Welfare (CA105-SP-01), the National Health Research Institutes (CA-105-PP-22), the Ministry of Science and Technology (MOST 105-2325-B-400-001; MOST-105-2320-B-400-011-MY3), and Ministry of Education (Aim for the Top University Project at National Cheng Kung University) of Taiwan.

\section{Availability of data and materials}

No applicable

\section{Authors' contributions}

JYC and CCK provided the conception and design the study. ITT performed the experiments. ITT and CCK analyzed data. CCK, ITT, and JYC wrote the main manuscript text. JPL Designed and synthesized MPTOB098. All authors read and approved the final manuscript.

\section{Ethics approval and consent to participate \\ No applicable}

\section{Consent for publication}

No applicable

\section{Competing interests}

The authors declare that they have no competing interests.

\section{Publisher's Note}

Springer Nature remains neutral with regard to jurisdictional claims in published maps and institutional affiliations.

\section{Author details}

'National Institute of Cancer Research, National Health Research Institutes, Tainan, Taiwan. ${ }^{2}$ Institute of Molecular Medicine, College of Medicine, National Cheng Kung University, Tainan, Taiwan. ${ }^{3}$ Institute of Biotechnology and Pharmaceutical Research, National Health Research Institutes, Zhunan, Taiwan. ${ }^{4}$ Institute of Clinical Pharmacy and Pharmaceutical Sciences, College of Medicine, National Cheng Kung University, Tainan, Taiwan. ${ }^{5}$ Graduate Program for Aging, China Medical University, Taichung, Taiwan. ${ }^{6}$ College of Pharmacy, Taipei Medical University, Taipei, Taiwan. ${ }^{7}$ Division of Hematology/ Oncology, Department of Internal Medicine, National Cheng Kung University Hospital, College of Medicine, National Cheng Kung University, Tainan, Taiwan.

\section{Received: 1 November 2017 Accepted: 24 March 2018}

\section{Published online: 28 March 2018}

\section{References}

1. Brown JM, Giaccia AJ. The unique physiology of solid tumors: opportunities (and problems) for cancer therapy. Cancer Res. 1998;58(7):1408-16.

2. Bennewith $\mathrm{KL}$, Dedhar $\mathrm{S}$. Targeting hypoxic tumour cells to overcome metastasis. BMC Cancer. 2011;11:504.

3. Ruas JL, Poellinger L. Hypoxia-dependent activation of HIF into a transcriptional regulator. Semin Cell Dev Biol. 2005;16(4-5):514-22.

4. Thiery JP. Epithelial-mesenchymal transitions in tumour progression. Nat Rev Cancer. 2002;2(6):442-54.

5. Guan X. Cancer metastases: challenges and opportunities. Acta Pharm Sin B. 2015;5(5):402-18.

6. Bredell MG, Ernst J, El-Kochairi I, Dahlem Y, Ikenberg K, Schumann DM. Current relevance of hypoxia in head and neck cancer. Oncotarget. 2016; 7(31):50781-804

7. Swartz JE, Pothen AJ, Stegeman I, Willems SM, Grolman W. Clinical implications of hypoxia biomarker expression in head and neck squamous cell carcinoma: a systematic review. Cancer Med. 2015;4(7):1101-16.

8. Brizel DM, Sibley GS, Prosnitz LR, Scher RL, Dewhirst MW. Tumor hypoxia adversely affects the prognosis of carcinoma of the head and neck. Int J Radiat Oncol Biol Phys. 1997;38(2):285-9.
9. Nordsmark M, Overgaard M, Overgaard J. Pretreatment oxygenation predicts radiation response in advanced squamous cell carcinoma of the head and neck. Radiother Oncol. 1996;41(1):31-9.

10. Peng HY, Cheng YC, Hsu YM, Wu GH, Kuo CC, Liou JP, Chang JY, Jin SL, Shiah SG. MPTOB098, a microtubule inhibitor, suppresses JAK2/STAT3 signaling pathway through modulation of SOCS3 stability in oral squamous cell carcinoma. PLoS One. 2016;11(7):e0158440.

11. Nien CY, Chen YC, Kuo CC, Hsieh HP, Chang CY, Wu JS, Wu SY, Liou JP, Chang JY. 5-Amino-2-aroylquinolines as highly potent tubulin polymerization inhibitors. J Med Chem. 2010;53(5):2309-13.

12. Cheng YC, Liou JP, Kuo CC, Lai WY, Shih KH, Chang CY, Pan WY, Tseng JT, Chang JY. MPTOB098, a novel microtubule inhibitor that destabilizes the hypoxia-inducible factor-1alpha mRNA through decreasing nuclearcytoplasmic translocation of RNA-binding protein HuR. Mol Cancer Ther. 2013;12(7):1202-12.

13. Yang $C Y$, Meng $C L$. Regulation of $P G$ synthase by EGF and PDGF in human oral, breast, stomach, and fibrosarcoma cancer cell lines. J Dent Res. 1994; 73(8):1407-15

14. Finlay GJ, Baguley BC, Wilson WR. A semiautomated microculture method for investigating growth inhibitory effects of cytotoxic compounds on exponentially growing carcinoma cells. Anal Biochem. 1984;139(2):272-7.

15. Pirozzi G, Tirino V, Camerlingo R, Franco R, La Rocca A, Liguori E, Martucci N, Paino F, Normanno N, Rocco G. Epithelial to mesenchymal transition by TGFbeta-1 induction increases stemness characteristics in primary non small cell lung cancer cell line. PLoS One. 2011;6(6):e21548.

16. Garg M. Epithelial-mesenchymal transition - activating transcription factors multifunctional regulators in cancer. World J Stem Cells. 2013;5(4):188-95.

17. Blanchoin L, Boujemaa-Paterski R, Sykes C, Plastino J. Actin dynamics, architecture, and mechanics in cell motility. Physiol Rev. 2014;94(1):235-63.

18. Stricker J, Falzone T, Gardel ML. Mechanics of the F-actin cytoskeleton. J Biomech. 2010;43(1):9-14.

19. Parsons JT, Martin KH, Slack JK, Taylor JM, Weed SA. Focal adhesion kinase: a regulator of focal adhesion dynamics and cell movement. Oncogene. 2000; 19(49):5606-13.

20. van Nimwegen MJ, van de Water B. Focal adhesion kinase: a potential target in cancer therapy. Biochem Pharmacol. 2007;73(5):597-609.

21. Mitra SK, Hanson DA, Schlaepfer DD. Focal adhesion kinase: in command and control of cell motility. Nat. Rev. Mol. Cell Biol. 2005;6(1):56-68.

22. Lamouille S, Xu J, Derynck R. Molecular mechanisms of epithelialmesenchymal transition. Nat. Rev. Mol. Cell Biol. 2014;15(3):178-96.

23. Morrison CD, Parvani JG, Schiemann WP. The relevance of the TGF-beta paradox to EMT-MET programs. Cancer Lett. 2013;341(1):30-40.

24. Chaudary N, Hill RP. Hypoxia and metastasis. Clin Cancer Res. 2007;13(7): 1947-9.

25. Escuin D, Kline ER, Giannakakou P. Both microtubule-stabilizing and microtubule-destabilizing drugs inhibit hypoxia-inducible factor-1alpha accumulation and activity by disrupting microtubule function. Cancer Res. 2005;65(19):9021-8.

26. Jung $H Y$, Fattet $L$, Yang J. Molecular pathways: linking tumor microenvironment to epithelial-mesenchymal transition in metastasis. Clin Cancer Res. 2015;21(5): 962-8.

27. Yang WH, Lan HY, Huang CH, Tai SK, Tzeng CH, Kao SY, Wu KJ, Hung MC, Yang MH. RAC1 activation mediates Twist1-induced cancer cell migration. Nat Cell Biol. 2012;14(4):366-74

28. Stehbens SJ, Akhmanova A, Yap AS. Microtubules and cadherins: a neglected partnership. Front Biosci (Landmark Ed). 2009;14:3159-67.

29. Yang MH, Wu MZ, Chiou SH, Chen PM, Chang SY, Liu CJ, Teng SC, Wu KJ. Direct regulation of TWIST by HIF-1alpha promotes metastasis. Nat Cell Biol. 2008;10(3):295-305.

30. Cheng JC, Klausen C, Leung PC. Hypoxia-inducible factor 1 alpha mediates epidermal growth factor-induced down-regulation of $\mathrm{E}$-cadherin expression and cell invasion in human ovarian cancer cells. Cancer Lett. 2013;329(2): 197-206.

31. Gonzalez DM, Medici D. Signaling mechanisms of the epithelial-mesenchymal transition. Sci Signal. 2014;7(344):re8.

32. Thiery JP, Sleeman JP. Complex networks orchestrate epithelial-mesenchymal transitions. Nat Rev Mol Cell Biol. 2006; (2):131-42.

33. Pu J, Zhang X, Luo H, Xu L, Lu X, Lu J. Adrenaline promotes epithelial-tomesenchymal transition via HuR-TGFbeta regulatory axis in pancreatic cancer cells and the implication in cancer prognosis. Biochem Biophys Res Commun. 2017:493(3):1273-9. 
34. Heldin CH, Landstrom M, Moustakas A. Mechanism of TGF-beta signaling to growth arrest, apoptosis, and epithelial-mesenchymal transition. Curr Opin Cell Biol. 2009;21(2):166-76.

35. Zhang H, Akman HO, Smith EL, Zhao J, Murphy-Ullrich JE, Batuman OA. Cellular response to hypoxia involves signaling via Smad proteins. Blood. 2003;101(6):2253-60.

36. Cicchini C, Laudadio I, Citarella F, Corazzari M, Steindler C, Conigliaro A, Fantoni A, Amicone L, Tripodi M, TGFbeta-induced EMT. Requires focal adhesion kinase (FAK) signaling. Exp Cell Res. 2008;314(1):143-52.

Submit your next manuscript to BioMed Central and we will help you at every step:

- We accept pre-submission inquiries

- Our selector tool helps you to find the most relevant journal

- We provide round the clock customer support

- Convenient online submission

- Thorough peer review

- Inclusion in PubMed and all major indexing services

- Maximum visibility for your research

Submit your manuscript at www.biomedcentral.com/submit
Biomed Central 